\title{
On-chip light diffraction imaging of nano structures in the guanine platelet
}

\author{
Masakazu Iwasaka \\ Hiroshima University \\ Kagamiyama 1-4-2 Higashihiroshima, Hiroshima 739-8527, Japan \\ Email: biomagnetics@siren.ocn.ne.jp
}

20 


\begin{abstract}
Light projection over short distances can minimize the size of photonic devices, e.g., headmounted displays and lens-free microscopes. Small lenses or light condensers without typical 10 lenses are essential for light control in micron-scale spaces. In this work, micro-platelets floating in water are used for light projection near the image sensor. These platelets, which are made from guanine, have nanohole gratings and demonstrate light diffraction toward specific directions. By setting a thin water layer on the image sensor's cover glass, each platelet in water forms columnor bar-code-shaped images on the screen. The projected image shapes and colors are inferred to contain information about nano-structures present in the guanine platelet. The proposed downsized imaging technique can realize extremely compact and portable imagers for nanoscale object detection.
\end{abstract}


The miniaturization of photonic devices is contributing to the diversity of the ubiquitous technology available in modern society. In portable devices such as mobile phone and small lifescience-oriented lens-free microscopes, charge-coupled devices (CCD) or complementary metaloxide-semiconductor (CMOS) image sensors are widely used. The latter sensors are used to perform on-chip imaging (1-3), which offers the advantage of realizing imaging of cells and living tissues within a compact space (4 -12).

The development of endoscopes and cell culture microscopes without lenses reduced the size of the apparatus required and its performance was improved by applying computational analysis. In the case of application to cellular imaging, microfluidic devices were shown to be effective in locating targeted cells close to the image sensor. For the illumination optics, the socalled shadow imaging method was applied with further image processing. Image reconstruction using holographic images and a deep learning approach $(11,12)$ were used to address the challenge of obtaining the same spatial resolution that is provided by a microscope with a lens.

However, additional potential remains for further miniaturization of the illumination optics. Utilization of planar films with nano-structures such as metasurfaces offers one potential approach for reducing the size of the illumination optics. $(13,14)$ Various types of flat optics structures were reported to act as functional lenses, thus achieving focusing without using a classical lens. (15-16) Nano-structures such as nanoholes (17-21) or grating (22) formed in a substrate have also achieved light focusing abilities.

Over the last two decades, studies of artificial nano-fabrication techniques for flat optics have opened up new ways to obtain flat lenses. In contrast, with regard to lenses in living systems, studies have continued to focus on classical dielectric lenses. There have been no examples of biological flat optics using similar mechanisms to those reported recently in optical material 25 science.

In the bio-photonics field, as a material responsible for control of biological light reflection, guanine platelets obtained from teleost fish skin have been investigated since the 1960s (23). The light reflecting properties of these platelets were explained (24) by considering the platelet to be a thin uniform platelet composed of guanine, which is one of the nucleic acid base molecules. Recently, scanning electron microscopy (SEM) and transmission electron microscopy (TEM) analyses of the guanine platelets of two species revealed that these platelets have sub- 
micrometer structures composed of triangular holes in each platelet (25) that were formed during or after their bio-crystallization process.

Figure 1 shows that optical microscopes can also visualize these sub-micrometer structures by tracking slight changes in the illumination conditions. In Fig. 1A, observation of the grating on the main face of a guanine platelet was achieved during a slight tilting motion of the platelet. Figure 1 and Figures S1-S4 indicate the existence of a grating with a width of less than $1 \mu \mathrm{m}$. During slight tilting of the tiny, thin platelet, images indicating the existence of nano-holes in the platelet were acquired. The platelet shown in Fig. 1B had holes arranged along the sides of triangular shapes, which corresponded to the triangular structure observed in the corresponding SEM image (Fig. 1D).

A

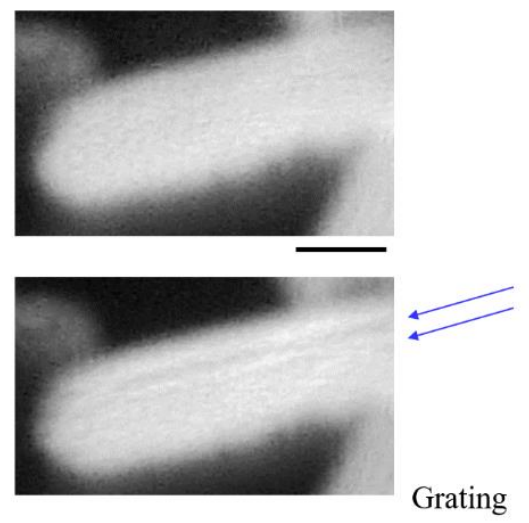

20

25
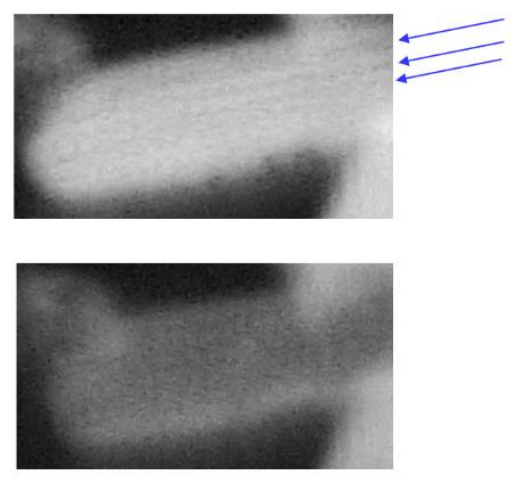

B
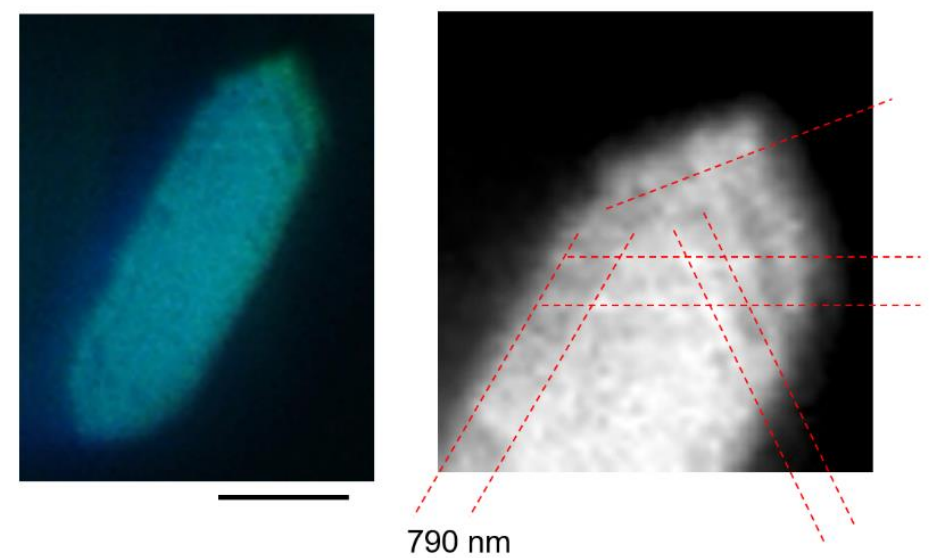

C

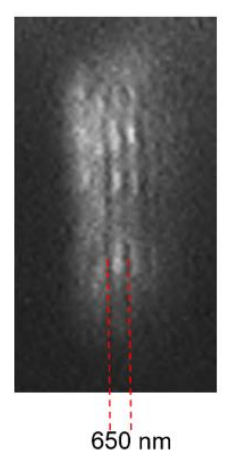

$790 \mathrm{~nm}$

D

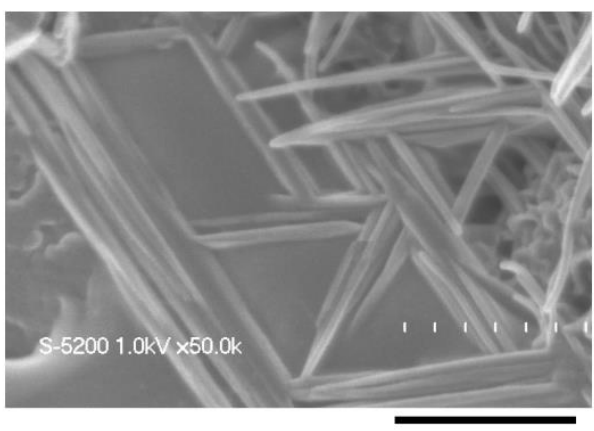

Figure 1. Grating structure observed in microscope images of fish guanine platelets. (A) Optical microscope images of a guanine platelet (main face) from a goldfish, Carassius auratus. The scale bar represents $5 \mu \mathrm{m}$. (B),-(C) Optical microscope images of guanine platelets from a Japanese anchovy, Engraulis japonicus. The scale bar represents $4 \mu \mathrm{m}$. (D) SEM image of the Japanese anchovy platelet. The scale bar represents $600 \mathrm{~nm}$. 
It was found that these fish guanine platelets in water caused strong contrast images to be observed on a CMOS image sensor array without a lens (26, submitted). In the improved imaging system shown in Fig. 2 (and Fig. S5), on-chip imaging was performed by directing the sensor array downward toward a black substrate, which acted to provide light shading. The length of gap between the image sensor and the black substrate was adjustable from $0.5 \mathrm{~mm}$ up to $2 \mathrm{~mm}$. A water layer containing the guanine platelets was then formed in this gap. The tilting angle of the guanine platelets in water was uncontrolled, which meant that various types of light scattering occurred on both the reflection side and the transmission side (Fig. 2A).

When the gap thickness was varied, column-shaped or bar-code-like images were obtained (Fig. 2C and 2D, respectively), along with the platelet-like projection image (Fig. 2B) on the image sensor (see Supplementary movie S1). It was thus possible, that the tiny platelets with sub-micrometer-scale holes contributed to the arrangements of the light scattering patterns appearing on the reflection side and the transmission side (Fig. 2E).

Apart from the three typical image types (Fig. 2B- D and Fig. S6- S10), other variant images appeared as an incidental case (see Fig. 3 and Fig. S11- 12). These colored, large-scale or assembled patterns involved column-type or bar-code-type components (see Supplementary movie S2). When compared with the typical patterns, these patterns were larger in size and there was a clear occurrence of light dispersion (Fig. 3A). The two large patterns that appear in Fig. 3A (top panel) individually caused a to-and-fro motion. This indicates that the projection originated from one object. These patterns were provably composed of complexes of platelets, such as stacked platelets. Because the platelets were obtained from fish scales, they are likely to be contaminated with biological substances (e.g., protein, lipids). Although, the variants shown in Fig. 2B were not colored, the patterns were composed of eight to nine bar-code shapes that moved together. A complex composed of two to three platelets floating in the water provably projected the patterns onto the image sensor plane. 
A
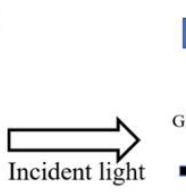

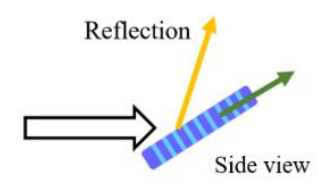

10

C
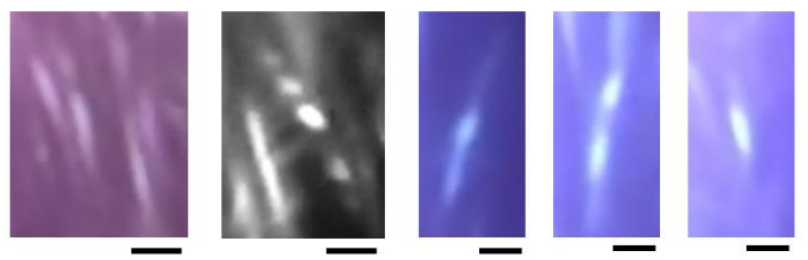

B

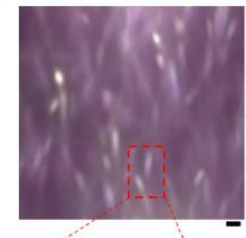

D
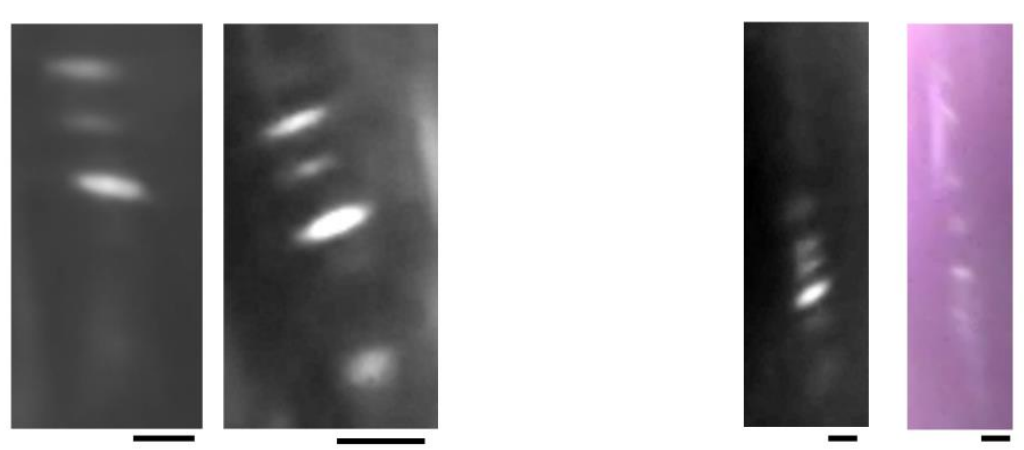

E

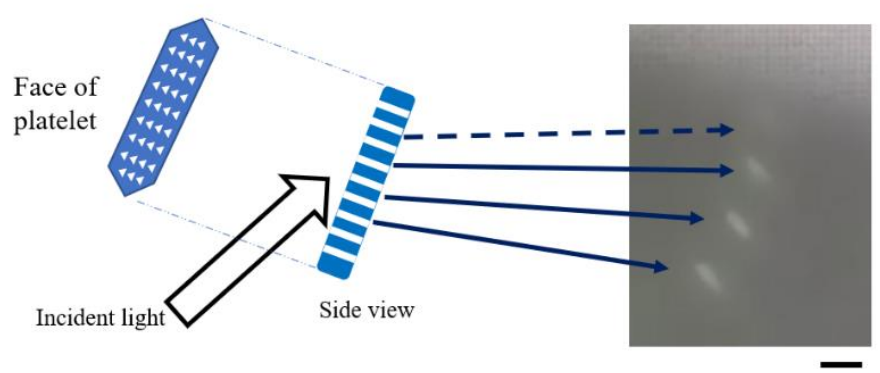

Figure 2. On-chip light scattering imaging of tiny goldfish platelets. (A) Scheme for imaging of platelets floating in water that form a thin layer beneath a CMOS sensor chip. The lower part of the illustration shows the speculated light scattering patterns. (B)- (D) Typical image patterns projected on the sensor array without the lens, where (B) shows the platelet-like pattern, (C) shows the column-type pattern, and (D) shows the bar-code-type pattern. (E) Speculated relationship between a platelet and projected images. The scale bar represents $100 \mu \mathrm{m}$. 


\section{A}

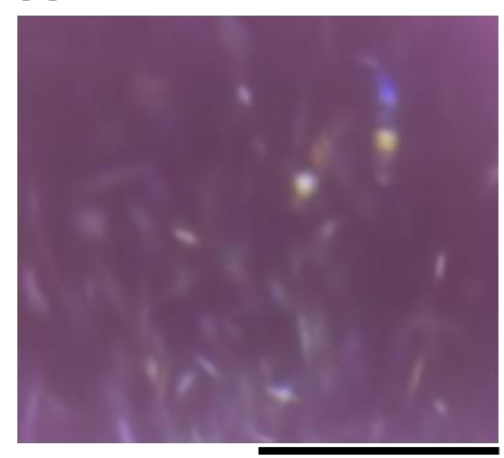

10

15
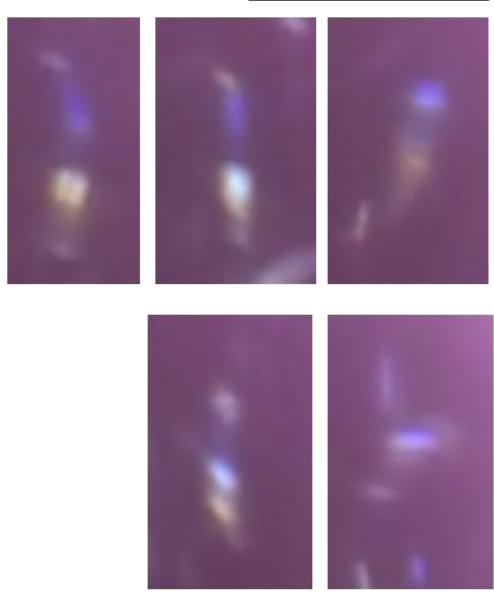

B

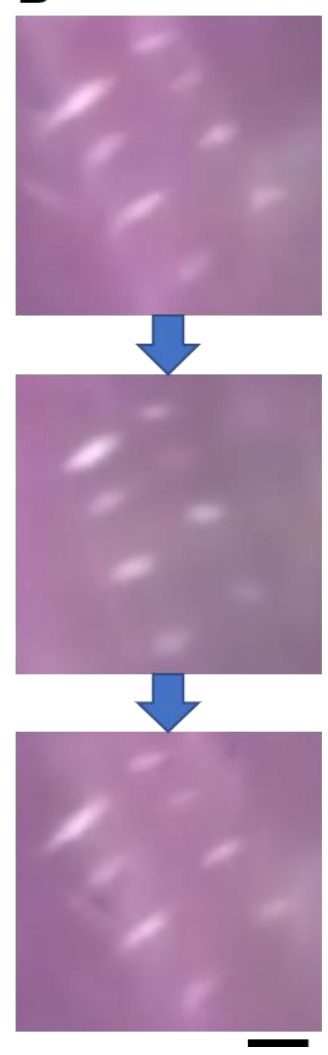

C

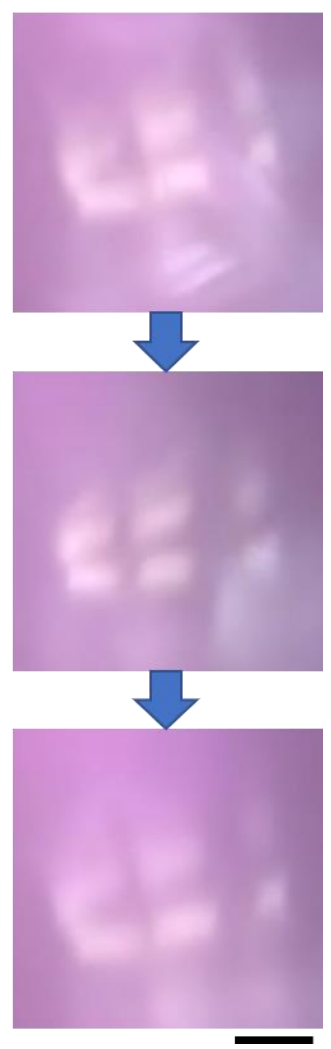

Figure 3. Variant light scattering patterns produced by guanine platelets from Japanese anchovy. A) Colored patterns appearing in a mass of images (top). The scale bar represents 1 $\mathrm{mm}$. The middle and bottom panels show five examples of the colored light scattering images.

B) Light scattering patterns from two connected platelets (left) and three connected platelets (right). The arrows indicate the time series of the changes in the image. The scale bar represents $100 \mu \mathrm{m}$. 
To clarify the light scattering patterns from the guanine platelets observed in the lens-free CMOS sensor array, a numerical simulation was performed using the finite-difference timedomain (FDTD) method (Fig. 4 and Fig. S13-22). Specifically, it was impossible to explain the generation of the bar-code pattern using regular reflections only. In fact, regular reflections (and forward scattering in the direction parallel to that of the incident light) provide the maximum intensity for the scattered light. It is thus necessary to find a comparable sub-branch among the scattering patterns. The dependence of the formation of the scattering pattern (logarithmic spherical plot) on the angle of incidence, the wavelength of the incident light, and the presence of the grating was thus investigated (Fig. 4A- C).

In Fig. 4A, the angle of incidence with respect to the main face (i.e., the broadest plane) of the platelet was $60^{\circ}$. When compared with a platelet without the grating (bottom panel of Fig. 4A), the platelet with the grating (middle panel of Fig. 4A) had more light scattering branches in the transmission side. Figure 4B shows the case where the main face of the platelet was oriented to be parallel to the incident light. It is obvious that the grating in the platelet was inducing the inclination in the vector of the light scattering normal to the main face. Strongly and highly-directionally scattered light was directed at angles of less than $\pm 30^{\circ}$ with respect to the direction normal to that of the incident light. This tendency became more distinct when the angle of incidence was $30^{\circ}$ (Fig. 4C). The scattering pattern was symmetrical, with reflection peaks and diffraction peaks existing in equal numbers. In addition to the reflection and diffraction peaks, a spherical plot of the scattered light in Fig. 4c shows that strong light (radiation-like) scattering occurred in the direction parallel to the platelet surface for light at wavelength of $500 \mathrm{~nm}$ and $630 \mathrm{~nm}$. The intensity of this strong and broad scattering behavior was similar to that of regular reflections. Figure 4D indicates that the unique scattering that occurred parallel to the platelet was related to the light propagation inside the 110-nm-thick platelet. The internal light propagation continued for 20 fs after the wave-front of the incident light passed through the platelet. The distances between the holes in the platelet simulation model were $540 \mathrm{~nm}$ and $270 \mathrm{~nm}$, as shown in the Supplementary Material. The light scattering pattern can be varied by changing the geometrical parameters of the platelet and the grating (holes). A platelet without a grating can also generate the diffraction branch in the light transmission side of the platelet. However, formation of the hole grating apparently 30 enhanced the intensity of the diffractions, which were related to the bar-code-type projection on the CMOS sensor. 
The results indicate that the fish guanine platelets used in this case acted as a diffractive lens (27). Unlike an artificial diffractive lens formed in a film on a substrate, the biogenic fish guanine platelets used in these experiments were initially formed in the organelles of a living cell and were then extracted and dispersed in an aqueous solution. Because the platelets can be floated individually in water, they can operate as a three-dimensional diffractive lens in a thin water layer.

A
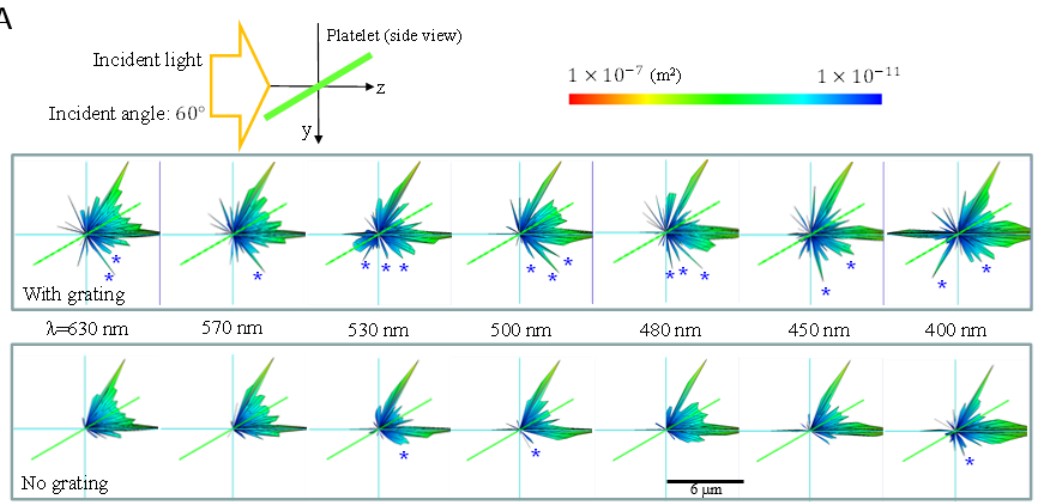

B

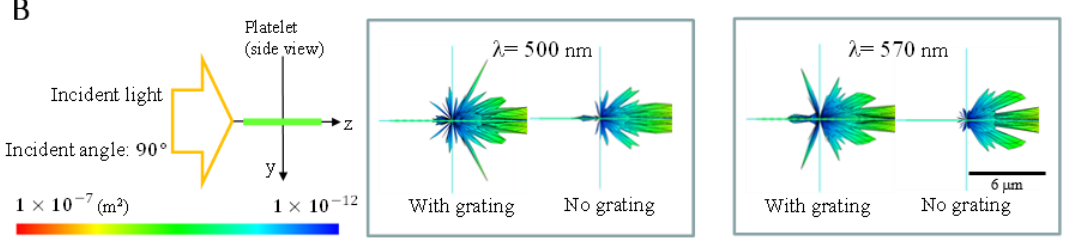

C

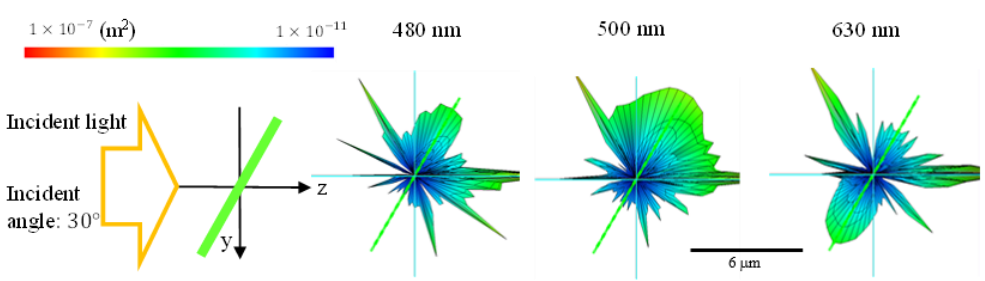

D
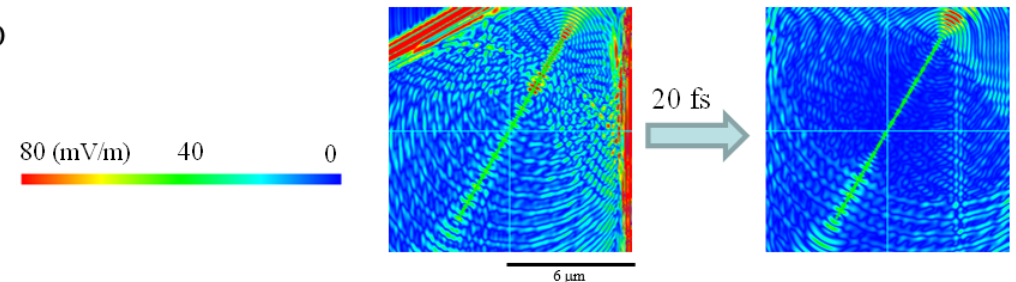

Figure 4. Electromagnetic FDTD simulation results of light scattering by a guanine platelet floating in water. The platelet contained a grating formed by an alignment of nano-holes filled with water. The refractive index of guanine was set at 1.83. (see the Supplementary Material for more detailed specifications of the simulation model). The incident light propagating along the $\mathrm{z}-$ axis was $s$-polarized when the incident plane was set to be the $\mathrm{y}$-z plane. A-C show spherical plots 
of the scattered light over the wavelength range from $630 \mathrm{~nm}$ to $400 \mathrm{~nm}$. (A) Comparison of light scattering by a guanine platelet with and without a grating when the angle of incidence was $60^{\circ}$. The blue asterisk marks a distinct branch of the scattered light appearing on the transmission side. (B) Light scattering by a guanine platelet when the angle of incidence was $90^{\circ}$ (for $\lambda=500 \mathrm{~nm}$ and $570 \mathrm{~nm}$ ). (C) Light scattering by a guanine platelet when the angle of incidence was $30^{\circ}$ (only for the platelet with the grating at $\lambda=480 \mathrm{~nm}, 500 \mathrm{~nm}$ and $630 \mathrm{~nm}$ ). (D) Electric field distribution around the platelet after the incident light passed through it. The color indicators indicate the crosssectional area $\left(\mathrm{m}^{2}\right)$ in (A)- (C) and the electric field in (D). The scale bar represents $6 \mu \mathrm{m}$.

The three basic three types of scattering pattern can be attributed to the structure of the individual platelets and the tilting angle versus the angle of light incidence. These simple image signals from the tiny platelets floating in water are suitable for detection of micro-environmental conditions. Imaging of both micromechanical motion and micro/nanoscale structures is possible when the guanine platelets are used as an on-chip reflection imaging transducer. We can make the angle of incidence higher, reduce the thickness of the illumination optics, and then use the advantages of the tiny, thin platelets, which not only offer strong reflection but also have diffraction lens properties. Other holographic-type on-chip imaging methods have also used the light diffraction patterns of the targeted object when the incident light was provided along the optical axis. In the on-chip imaging process in this work, only the fish guanine platelets exhibited images with strong contrast, while other particles suspended in the water did not show images with the same contrast properties.

If the colorful variants shown in Fig. 3 were the product of stacked platelets, the variant image should then be a consequence of the interactions between the tiny platelets and other objects. A component with a bar-code pattern originated from a guanine platelet with dimensions of 20$40 \mu \mathrm{m} \times 5-10 \mu \mathrm{m} \times 100-160 \mathrm{~nm}$. Therefore, the emergence of the variants (as shown in Fig. 3B) indicates integration of the platelets. For future applications of on-chip light diffraction imaging with fish guanine platelets, use of additional image analysis methods such as deep learning techniques may be effective. 


\section{References}

1. S. O. Isikman, W. Bishara, S. Mavandadi, F. W. Yu, S. Feng, R. Lau, A. Ozcan, Lens-free optical tomographic microscope with a large imaging volume on a chip, Proc Natl Acad Sci U $S$ A., 108(18), 7296-7301 (2011).

2. A. Greenbaum, W. Luo, T. W. Su, Z. Göröcs, L. Xue, S. O. Isikman, A. F. Coskun, O. Mudanyali, A. Ozcan, Imaging without lenses: achievements and remaining challenges of wide-field on-chip microscopy, Nat Methods, 9(9), 889-895 (2012)

3. Y. Zhang, Y. Wu, Y. Zhang, A. Ozcan. Color calibration and fusion of lens-free and mobilephone microscopy images for high-resolution and accurate color reproduction. Sci Rep., 6 , 27811 (2016).

4. J. Shin, D. N. Tran, J. R. Stroud, S. Chin, T. D. Tran, M. A. Foster, A minimally invasive lensfree computational microendoscope. Sci Adv., 5(12), eaaw5595 (2019) doi: 10.1126/sciadv.aaw5595

5. Y. Zhang, Y. Shin, K. Sung, S. Yang, H. Chen, H. Wang, D. Teng, Y. Rivenson, R. P. Kulkarni, A. Ozcan, 3D imaging of optically cleared tissue using a simplified CLARITY method and onchip microscopy. Sci Adv. 3(8), e1700553 (2017) doi: 10.1126/sciadv.1700553

6. E. Mathieu, C. D. Paul, R. Stahl, G. Vanmeerbeeck, V. Reumers, C. Liu, K. Konstantopoulos, L. Lagae, Time-lapse lens-free imaging of cell migration in diverse physical microenvironments. Lab Chip 16(17):3304-3316 (2016) doi: 10.1039/c6lc00860g.

7. J. Kun, M. Smieja, B. Xion, L. Soleymani, Q. Fang, The Use of Motion Analysis as Particle Biomarkers in Lensless Optofluidic Projection Imaging for Point of Care Urine Analysis, Sci Rep. 9(1):17255 (2019) doi: 10.1038/s41598-019-53477-8.

8. R. Ramji, C. F. Cheong, H. Hirata, A. R. Rahman, C. T. Lim, Rapid quantification of live cell receptors using bioluminescence in a flow-based microfluidic device, Small, 11(8), 943-951 (2015) doi: 10.1002/smll.201401674

9. Y. Fang, N. Yu, R. Wang, D. Su, An on-chip instrument for white blood cells classification based on a lens-less shadow imaging technique, PLoS One, 12(3), e0174580 (2017) doi: 10.1371/journal.pone.0174580 
10. A. Ray, M. A. Khalid, A. Demčenko, M. Daloglu, D. Tseng, J. Reboud, J. M. Cooper, A. Ozcan, Holographic detection of nanoparticles using acoustically actuated nanolenses, Nat Commun 11(1), 171 (2020) doi: 10.1038/s41467-019-13802-1

11. S. J. Kim, C. Wang, B. Zhao, H. Im, J. Min, H. J. Choi, J. Tadros, N. R. Choi, C. M. Castro,

R. Weissleder, H. Lee, K. Lee, Deep transfer learning-based hologram classification for molecular diagnostics. Sci Rep. 8(1), 17003 (2018) doi: 10.1038/s41598-018-35274-x

12. Z. Göröcs, M. Tamamitsu, V. Bianco, P. Wolf, S. Roy, K. Shindo, K. Yanny, Y. Wu, H. C. Koydemir, Y. Rivenson, A. Ozcan, A deep learning-enabled portable imaging flow cytometer for cost-effective, high-throughput, and label- free analysis ofnatural water samples. Light Sci Appl. 7 (2018)

13. A. V. Kildishev, A. Boltasseva, V. M. Shalaev, Planar photonics with metasurfaces, Science 339, 1232009 (2013) doi: 10.1126/ Science.1232009

14. Y. Nanfang, C. Federico, Flat optics with designer metasurfaces, Nat. Mater. 13, 139-150 (2014) doi: 10.1038/NMAT3839

15. F-M. Huang, T-S. Kao, V. A. Fedotov, Y. Chen, N. I. Zheludev, Nanohole Array as a Lens, Nno Letters, 8(8), 2469-2472 (2008)

16. S. Ishii, V. M. Shalaev, A. V. Kildishev, Holey-Metal Lenses: Sieving Single Modes with Proper Phases, Nano Lett. 13, 159-163 (2013) doi:10.1021/n1303841n

17. C. Genet, T. W. Ebbesen, Light in tiny holes, Nature, 445 (2007) doi:10.1038/nature05350

18. J. He, P. Ding, J. Wang, C. Fan, E. Liang. Double Fano-type resonances in heptamer-hole array transmission spectra with high refractive index sensing. Journal of Modern Optics, 62 (15), 1241-1247 (2015) doi: 10.1080/09500340.2015.1030351

19. T. Kao, Y. Lo, H-C. Kuo. Imaging Functions of Quasi-Periodic Nanohole Array as an UltraThin Planar Optical Lens. Photonics 2(2), 619-633 (2015) doi: 10.3390/photonics2020619

20. Y-J. Liu, H. Liu, E-S-P. Leong, C-C. Chum, J-H. Teng, Fractal Holey Metal Microlenses with Significantly Suppressed Side Lobes and High-Order Diffractions in Focusing, Advanced Optical Materials 2 (5), 487-492 (2014) doi: 10.1002/adom.201300447 
21. J-C. Yang, H. Gao, J-Y. Suh, W. Zhou, M-H. Lee, T. W. Odom, Enhanced Optical Transmission Mediated by Localized Plasmons in Anisotropic, Three-Dimensional Nanohole Arrays. Nano Letters 10(8) , 3173-3178 (2010) doi: 10.1021/nl102078j

22. D. Fattal, J. Li, Z. Peng, M. Fiorentino, R. G. Beausoleil, Flat dielectric grating reflectors with focusing abilities, Nature Photon. 4, 466-470 (2010) doi: 10.1038/nphoton.2010.116

23. E. J. Denton, Review Lecture: On the organization of reflecting surfaces in some marine animals. Philos. Trans. R. Soc. London Ser. B 258, 285-313 (1970)

24. M. Iwasaka, H. Asada, Floating photonic crystals utilizing magnetically aligned biogenic guanine platelets. Scientific Reports, 8, 16940 (2018).

25. M. Iwasaka, Hidden triangular grating structures in biogenic guanine platelet, AIP Advances, 10 (9), 095133 (2020).

26. M. Iwasaka, bioRxiv doi: 10.1101/2021.04.03.438308

27. K. Huang, F. Qin, H. Liu, H. Ye, C-W. Qiu, M. Hong, B. Luk'yanchuk, J. Teng. Planar Diffractive Lenses: Fundamentals, Functionalities, and Applications. Advanced Materials 30 (26), 1704556 (2018) https://doi.org/10.1002/adma.201704556

Acknowledgments: The author thanks David MacDonald, MSc, from Edanz (https://jp.edanz.com/ac) for editing a draft of this manuscript.

Funding: JST-CREST “Advanced core technology for creation and practical utilization of innovative properties and functions based upon optics and photonics" (Grant number: JPMJCR16N1).

Author contributions: MI performed the experimental design, experiments, measurements and analyses. All parts of the manuscript and illustrations were prepared by MI.

Competing interests: The author declares no competing interests. materials. 